Tohoku J. exp. Med., 1971, 107, 149-157

\title{
Effect of Deoxycholate on Smooth-surface Membranes
}

\author{
Makoto SeiJi and Hideko Itakura \\ Department of Dermatology, Tohoku University School of \\ Medicine, Sendai
}

Seisr, M. and ITakura, H. Effect of Deoxycholate on Smooth-surface Membranes. Tohoku J. exp. Med., 1972, 107 (2), 149-157 — The effect of deoxycholate (DOC) on smooth-surface membranes was studied by means of electron microscopy. The smooth-surface membranes were isolated from HardingPassey mouse melanomas and treated with $0.05 \%$ DOC. In the sectioned specimens, the untreated smooth-surface membranes appear as unit membranebound, closed vesicles. The unit membranes measure $50 \AA$ across. The treated membranes showed significant vesiculation and swelling. In some parts many small vesicles are grouped in a cluster and enclosed by a membrane. A few to several unit membranes are stuck together to form myelin-like structures. In the negatively stained specimens, varying degrees of smooth-surface membrane vesiculation are apparent. Most large vesicles are found to exhibit myelin-like lamellar structures. From these observations, DOC appears to cause a vesiculation and swelling of the smooth-surface membranes but does not appear to destroy the unit membrane structure. There were a few myelin-like lamellar structures which were thought to have been produced by uniting several unit membranes. — deoxycholate; smooth-surface membranes; negatively stained electron microscopy

Although the exact mechanisms of surface active agents on the cytoplasmic membranes are not known, various detergents such as deoxycholate and cholate have been extensively used for the microdissection of mitochondria (Arion and Racker 1970) and for the preparation of the cytoplasmic organelles (Siekevitz and Palade 1966).

The status of tyrosinase has been studied by treating the smooth-surface membranes isolated from Harding-Passey mouse melanoma with deoxycholate (DOC) (Seiji and Iwashita 1964). It was revealed that about $95 \%$ of the total tyrosinase activity were found in the soluble phase with the presence of $0.4 \% \mathrm{DOC}$. When DOC was removed by gel filtration, however, a considerable amount of membranous material was obtained after centrifugation, and most of the tyrosinase activity, total protein, and phospholipids as well as other enzyme activities were thus recovered in the membranous sediment obtained. From these experimental results it was clear that the "solubilization" of tyrosinase and other constituents of the membranes by the DOC treatment was only apparent and could be reversed by the removal of DOC.

The experiment described in the present paper was performed in an effort to

Received for publication, November 29, 1971. 
clarify the effect of DOC on the smooth-surface membranes isolated from Harding-Passey mouse melanoma. Smooth-surface membranes were treated with $0.05 \%$ DOC and subjected to electron microscopic observation. The sectioned specimens as well as negatively stained specimens were studied.

\section{Materials and Methods}

\section{Preparation of the smooth-surface membranes}

Harding.Passey mouse melanomas, which had been serially transplanted in Swiss strain mice, were excised when they were about 1.0 to $1.5 \mathrm{~cm}$ in diameter and were promptly homogenized in nine volumes of $0.25 \mathrm{M}$ sucrose at $0^{\circ} \mathrm{C}$ with a Potter glass Teflon homogenizer to give a $10 \%$ homogenate ( $\mathrm{wt} / \mathrm{vol}$ ). All subsequent procedures were carried out at temperatures below $4^{\circ} \mathrm{C}$. The homogenate was centrifuged at $11,000 \times \mathrm{g}$ for $10 \mathrm{~min}$ and the resulting supernatant was centrifuged at $105,000 \times \mathrm{g}$ for $60 \mathrm{~min}$. The sediment was resuspended in $1.32 \mathrm{M}$ sucrose and the smooth-surface membrane fraction was isolated by the Rothschild method (Rothschild 1961) with a slight modification: the small granule suspension instead of the "mitochondrial supernatant" was placed at the bottom of the centrifuge tube.

\section{DOC treatment}

DOC in $0.25 \mathrm{M}$ sucrose and $0.05 \mathrm{M}$ Tris buffer, $\mathrm{pH} 7.8$, was mixed with the smoothsurface membrane suspension in $0.1 \mathrm{M}$ phosphate buffer, $\mathrm{pH} 7.0$, for a final concentration of $0.05 \%$. The protein content was determined according to Lowry et al. (1951) using bovine serum albumin as a standard.

\section{Morphological methods}

For electron microscopy, suitable aliquots of the suspension of the smooth-surface membranes were centrifuged at $105,000 \times \mathrm{g}$ for $\mathrm{l}$ hour. The sediment thus obtained was fixed at $0^{\circ} \mathrm{C}$ with $2.5 \%$ glutaraldehyde in phosphate buffer at pH 7.2 for 2 hrs and then in $1 \%$ osmium tetroxide solution for $2 \mathrm{hrs}$. The sediment was then dehydrated in a graded series of ethyl alcohol and embedded in Epon 812. The specimens were sectioned with a Porter-Blum Ultrathin microtome and stained doubly with uranyl acetate and lead citrate. The suspension of the smooth-surface membranes was also stained negatively with phosphotungstate using the method described by Oda and Nishi (1963).

\section{Results and Discussion}

Under the electron microscope, smooth-surface membranes appear as membrane-bound closed vesicles, as shown in Fig. 1. The membrane appears sectioned normally at $\mathrm{A}$, obliquely at $\mathrm{B}$, and grazingly at $\mathrm{C}$. These sections show the characteristic unit membrane appearance. It is noted that most smooth-surface membranes are organized in closed vesicles and that membrane fragments with free margins occur only in small numbers. Some of the vesicles seem to contain part of their content. A unit membrane measured $50 \AA$ across. The nature of the highly electron-dense small particles, some of which are attached to the inside surface of the vesicles, is not known at the present time yet, but they do not seem to be artifacts. As shown in the insert in Fig. 1, it is possible to see a figure in which two unit membranes are united side by side. Figure 2 shows an electron micrograph of smooth-surface membranes treated with $0.05 \%$ DOC. 
There are numerous small vesicles, 300 to $500 \AA$ in diameter, and some of them are grouped in a cluster and enclosed by a membrane, as shown in Fig. 3 . In some parts of the section there are large vesicles that appear to be swelling. Figure 4 shows that the unit membrane structure of $50 \AA$ in diameter appears to be retained, and a few to several unit membranes unite side by side to form a myelin-like lamellar structures.

Figs. 5 and 6 show electron micrographs of negatively stained smooth-surface membranes. The findings revealed in these micrographs are quite similar to those obtained in the sectioned specimens. Varying degrees of vesiculation of the smooth-surface membranes are apparent. Multivesicular body-like structures are also clearly seen. The repeating small particles, which were repeatedly seen on the untreated smooth-surface membranes in the negatively stained specimens, are not clearly visible (Seiji 1967). Most of the large vesicles are found to exhibit myelin-like lamellar structures. Since the negatively stained preparation only shows surface images of the objects, the lamellar structure seen in both specimens does not necessarily indicate the same membrane architecture. It does not seem unreasonable to assume that only certain tangentially sectioned vesicles show such figures in sectioned specimens and that they are multilayered externalcompound membranes which are formed by uniting the unit membranes (Robertson 1964). On the other hand, the lamellar structures seen in the negatively stained specimens are actually images of the regular lamellae formed on the surface of the vesicles. How such lamellar structures are formed on the surface of the vesicles, and why the multilayered external-compound membranes are formed by the DOC treatment, are not known. The lamellar structures seen in the negatively stained specimens do not seem to be the same as those forme 1 in the smooth-surface membranes after trypsin digestion (Seiji et al. 1971) but they closely resemble structures seen in electron micrographs of artificial lipid spherulss which are formed with ovolecithin, dicetyl phosphate and cholesterol (70:20:10 by weight, see Sessa and Weissman 1968).

\section{Acknowledgment}

This work was supported by research grants from the Ministry of Education, Japan, from Westwood Pharmaceuticals company, Buffalo, New York, from the Japan O'Leary Pigment Research Fund Osaka, and from the Takeda Science Foundation, Osaka.

\section{References}

1) Arion, W.J. \& Racker, E. (1970) Partial resolution of the enzyme catalyzing oxidative phosphorylation. XXIII. Preservation of energy coupling in submitochondrial particles lacking cytochrome oxidase. J. biol. Chem., 245, 5186-5194.

2) Lowry, O.H., Rosebrough. N.U., Farr, A.L. \& Randall, R.J. (1951) Protein measurement with the Folin phenol reagent. J. biol. Chem., 193, 265-275.

3) Oda, T. \& Nishi, Y. (1963) Fundamental structure and function of mitochondrial membrane. J. Electron Microscopy., 12, 290-295.

4) Rothschild, J.A. (1961) Sub-fractionation of rat liver microsomes. Fed. Proc., 20, 145. 
5) Seiji, M. \& Iwashita, S. (1964) The status of tyrosinase in smooth-surfaced membranes of melanocyte. J. Biochem., 56, 197-199.

6) Seiji, M. (1967) Subcellular particles and melanin formation in melanocytes. In "Advances in Biology of Skin", vol. VIII, The Pigmentary System, ed. by W. Montagna and F. Hu, Pergamon Press, Oxford and New York, pp. 189-222.

7) Seiji, M., Itakura, H. \& Irimajiri, T. (1972) Tyrosinase in the membrane system of mouse melanoma. In Pigmentation: Its Genesis and Biologic Control, ed. by V. Riley, Applton-Century-Crofts, New York, in press.

8) Sessa, G. \& Weissmann, G. (1968) Phospholipid spherules (liposomes) as a model for biological membranes. J. Lipid Res, 9, 310-318.

9) Siekevitz, P. \& Palade, G.E. (1966) Distribution of newly synthesized amylase in microsomal subfractions of guinea pig pancreas. $J$. Cell Biol., 30, 519-530.

Fig. 1. Section through a pellet of the untreated smooth-surface membranes isolated from Harding.Passey mouse melanoma. The pellet consists mainly of swollen smoothsurface membranes which have retained membranes and part of their contents. The membrane appears sectioned normally at $\mathrm{A}$, olbiquely at $\mathrm{B}$, and grazingly at $\mathrm{C}$. $\mathrm{A}$ unit membrane measures $50 \AA$ across. Magnification: $80,000 \times$, insert $200,000 \times$

Fig. 2. Section through a pellet of the DOC-treated smooth-surface membranes. There are numerous small vesicles, 300 to $500 \AA$ in diameter, and some of them are grouped in a cluster and enclosed by a membrane. $80,000 \times$. 


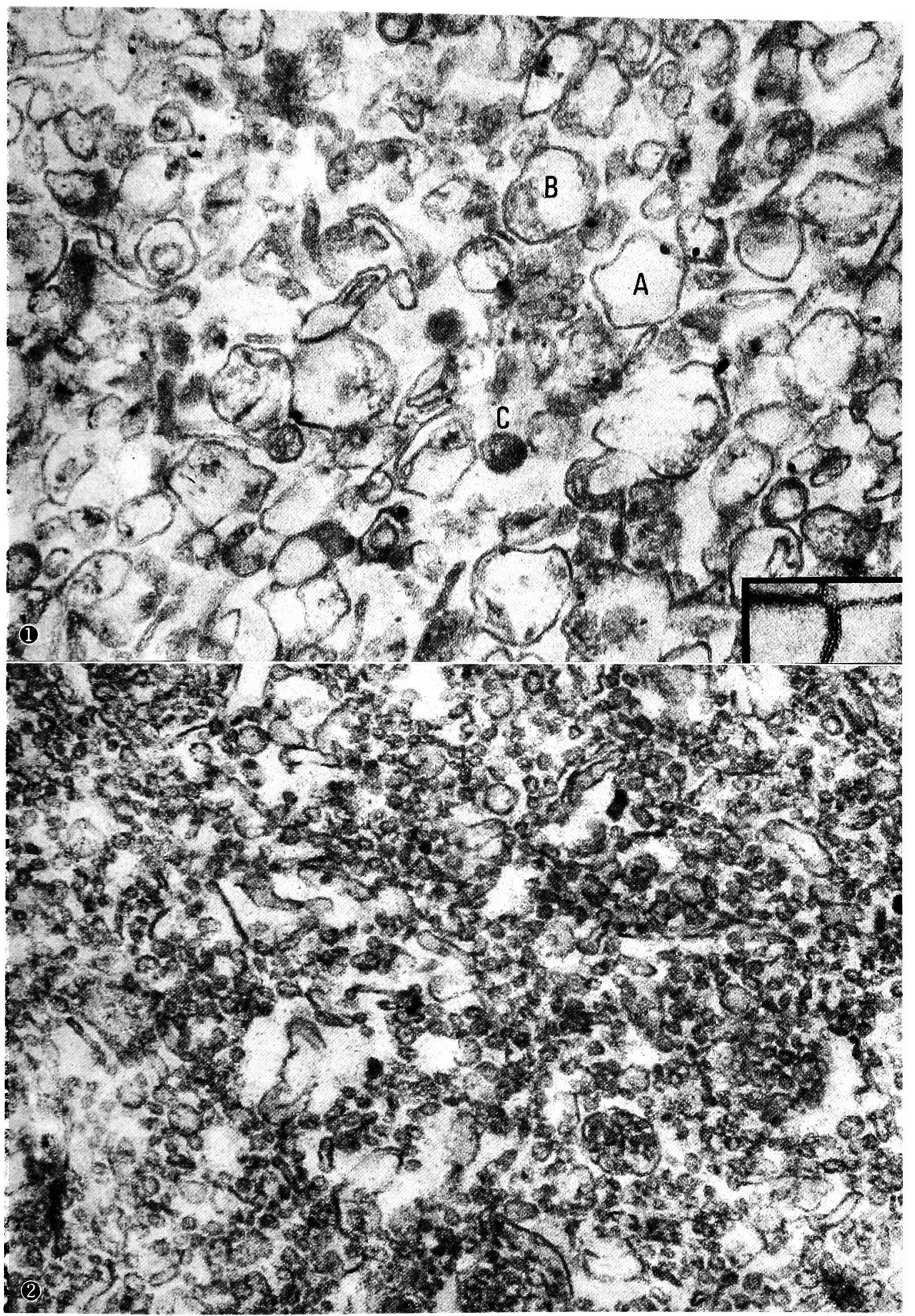


Fig. 3. Section through a pellet of the DOC-treated smooth-surface membrane. In some parts there are large swollen vesicles and a few multivesicular body-like structures. The unit membrane structure, $50 \AA$ in diameter, appears to be retained and a few to several unit membranes unite side by side to form myelin-like lamellar structures. $80,000 \times$.

Fig. 4. High magnification of part of a section through a pellet of the DOC-treated smoothsurface membrane. There are many small vesicles and a myelin-like lamellar structure. $200,000 \times$. 


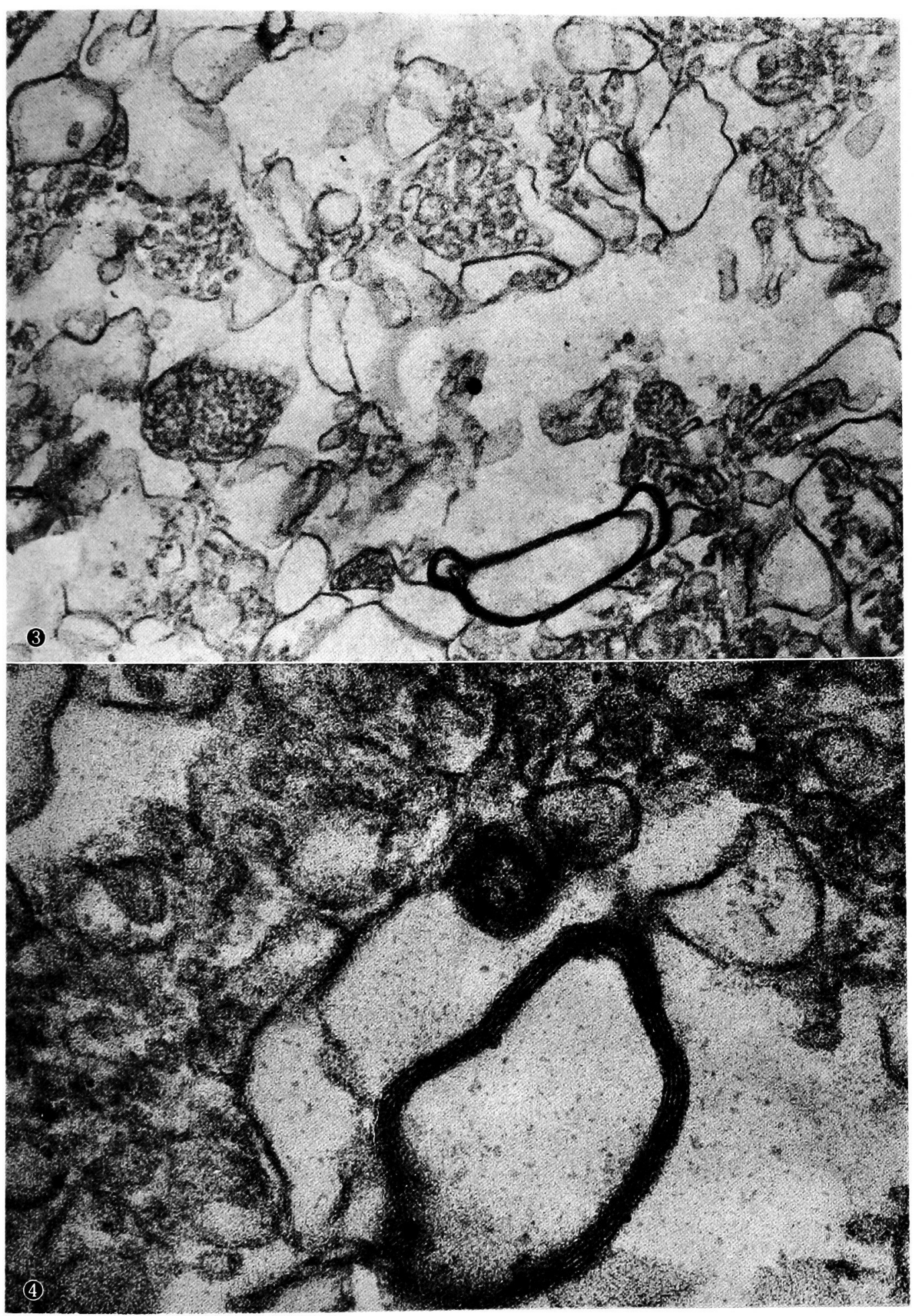


Fig. 5. Eelectron micrograph of the DOC-treated smooth-surface membranes isolated from Harding -Passey mouse melanoma and stained negatively with phosphotungstate. Many small vesicles of various sizes and multivesicular body-like structures are also visible. The large vesicles are found to exhibit myelin-like lamellar structures. $200,000 \times$.

Fig. 6. A part of the DOC-treated smooth-surface membranes stained negatively. There are lamellar structures on the outer and inner sides of the large veiscle. They seem to be regular lamellae formed on the surface of the veiseles. $200,000 \times$. 


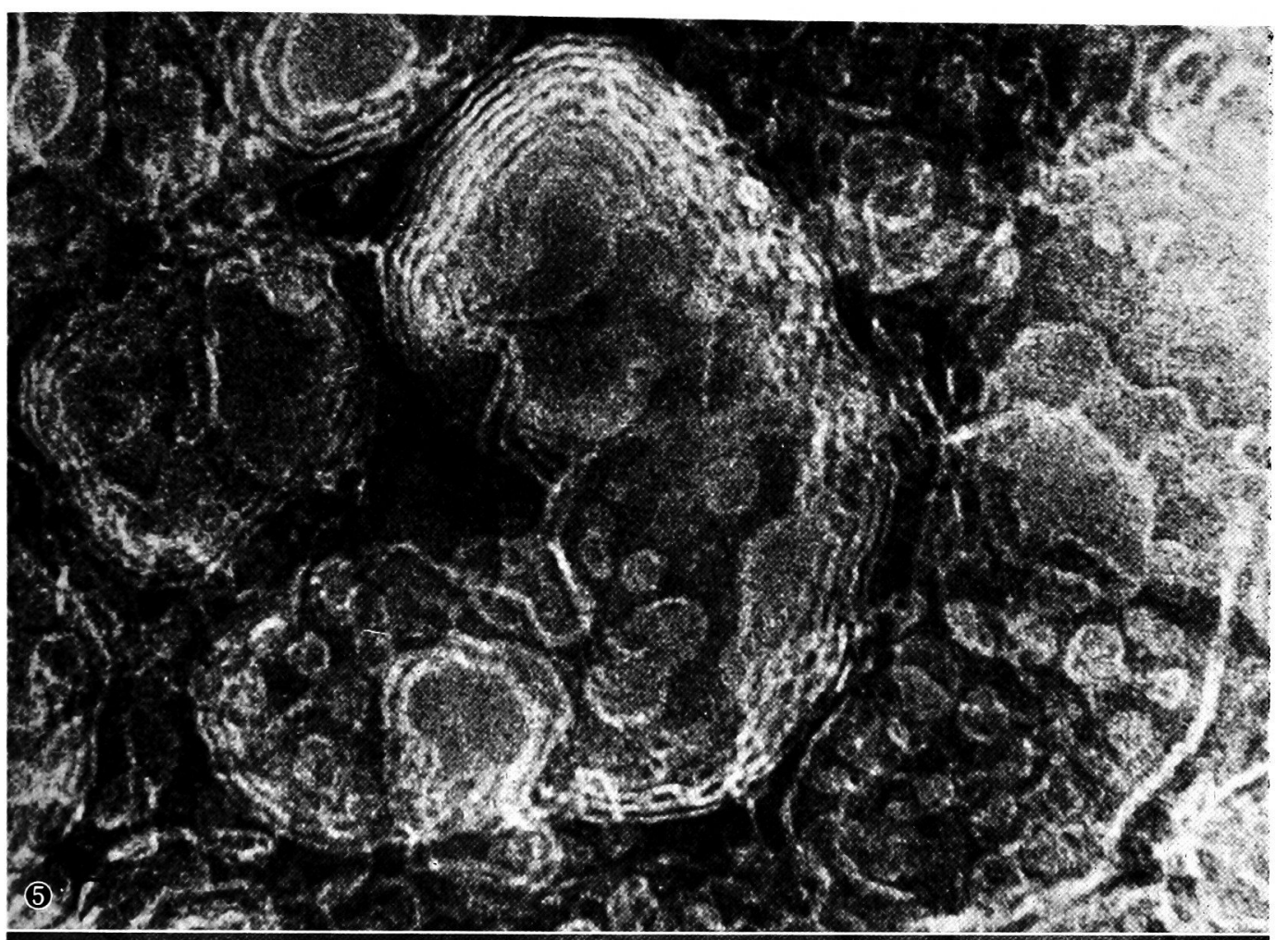

